\title{
Vínculos entre Ética, Tecnología Educativa y Pedagogía Hospitalaria: una revisión sistematizada de la literatura
}

\author{
Connecting Ethics, Educational Technology and Hospital Pedagogy: \\ a Systematic Review of Literature \\ D Martín García-Parra; m.garcia@uib.es \\ Marina Pérez Sepulcre; marina20.595@gmail.com \\ Universitat de les Illes Balears (España)
}

\begin{abstract}
Resumen
Vincular Ética, Tecnología Educativa y Pedagogía Hospitalaria se convierte en un reto interdisciplinar que pretende dar una respuesta comprometida e integral a necesidades educativas y biopsicosociales de niños, niñas, adolescentes y jóvenes hospitalizados. El objetivo del artículo es analizar y cartografiar posibles vínculos transversales que fomenten compromisos éticos interprofesionales, a través del uso de la Tecnología Educativa y la formación de la competencia digital docente, en el ámbito de la Pedagogía Hospitalaria. El método utilizado fue una revisión sistematizada de la literatura siguiendo las directrices de la Declaración PRISMA. Los resultados muestran cuatro dimensiones con experiencias y estudios que relacionan la ética, la Tecnología Educativa y la Pedagogía Hospitalaria. En conclusión, ante el reto de lograr una inclusión educativa integral y una mayor atención a la diversidad, es menester forjar un ethos interprofesional que utilice herramientas y recursos de la Tecnología Educativa para fomentar experiencias de aprendizaje enriquecedoras para niñas, niños, adolescentes o jóvenes hospitalizados.
\end{abstract}

Palabras clave: Ética, Tecnología Educativa, Pedagogía Hospitalaria, Inclusión Educativa, Revisión Sistematizada

\begin{abstract}
Connecting Ethics, Educational Technology and Hospital Pedagogy becomes an interdisciplinary challenge that aims to provide a committed and integral response to the educational and biopsychosocial needs of hospitalized children and young people. The goal of this paper is analysing and mapping possible connection that promote interprofessional ethical commitments, by Educational Technology and the training of teaching digital competence, in the field of Hospital Pedagogy. Method: a systematic review of the literature following the guidelines of the PRISMA Declaration. Results indicates four dimensions with experiences and studies that enable relationships between ethics, Educational Technology and Hospital Pedagogy. In sum, faced with the challenge of achieving an integral educational inclusion and an attention to diversity, it is necessary build an interprofessional ethos that uses tools and resources from Educational Technology for promote learning experiences for hospitalized children and youth.
\end{abstract}

Keywords: Ethics, Educational Technology, Hospital Pedagogy, Educational Inclusion, Systematic Review. 


\section{INTRODUCCIÓN}

Una educación humanista y holística es vital para hacer frente al reto de la inclusión de las personas más vulnerables que frecuentemente son discriminadas. Estas bases permiten un aprendizaje abierto y flexible que brinde a todos la oportunidad de realizar su potencial con miras a un futuro sostenible y una existencia digna. Además, la importancia de una educación para todos es mayor a causa del desarrollo de las nuevas tecnologías digitales. (UNESCO, 2015). Por ello, la ética surge como disciplina dialogante que afronta el reto inclusivo en diversos escenarios vulnerables fomentando buenas actuaciones relacionadas con la Pedagogía Hospitalaria (PH) y la Tecnología Educativa (TE). Sobre estas afirmaciones se impone la pregunta de investigación: ¿Cómo potenciar vínculos entre la ética, la Tecnología Educativa y la Pedagogía Hospitalaria para fomentar inclusión educativa? La pregunta de investigación suscita una apuesta por forjar en $\mathrm{PH}$ un carácter moral comprometido, crítico y consciente de que el uso de TE permite experiencias y aprendizajes significativos.

El vocablo griego ethos es el origen de la ética. El ethos "es un modo habitual, continuo, de comportarse, de ser en el tiempo; forma de estabilidad y persistencia temporal" (Gónzalez, 1996, p. 10). El ser humano es un sujeto moral por naturaleza que forja un carácter o modo de afrontar, por hábito y voluntad propia, la vida (Domingo, 2008). Por tal, un ethos profesional en $\mathrm{PH}$ es un carácter o modo de comportarse, de manera comprometida, para con el alumnado y su familia en un entorno hospitalario (García, 2019).

En relación con el ámbito tecnológico, en la actualidad, "moverse y orientarse en el ciberespacio no sólo implica el domino de ciertas competencias digitales, nivel de uso, sino que también supone la adquisición y desarrollo de competencias éticas, nivel de sentido" (GarcíaGutiérrez, 2013, p. 9). Así, el carácter ético también supone el desarrollo de la competencia digital docente, entendida como el conjunto de conocimientos, habilidades y actitudes para que una persona aprenda el uso de herramientas digitales, diseñe contenidos digitales que respondan a necesidades del alumnado y resuelva problemas en un entorno digital (Carretero et al., 2017; Ferrari, 2012).

Sobre la base de la PH, la inclusión educativa es el "proceso transformador que asegura la plena participación y el acceso a oportunidades de aprendizaje de calidad para todos los niños, niñas, jóvenes y adultos, que respeta y valora la diversidad, y elimina todas las formas de discriminación"(UNESCO, 2019, p. 1) Asimismo, asegurar el acceso a una educación de calidad es reconocer el valor de la diversidad y el respeto de la dignidad humana (Ainscow, 2019).

Violant et al. (2011), definen la PH como:

La acción pedagógica que se desarrolla durante los procesos de enfermedad para dar respuesta a las necesidades biopsicosociales derivadas de dicha situación, con el fin de mejorar el bienestar y la calidad de vida, garantizando los derechos con relación a la función educativa. (2011, p. 89).

Siguiendo la Carta Europea de los Derechos del niño hospitalizado (Carta Europea de Los Derechos Del Niño Hospitalizado, 1986) el énfasis principal de la PH es la educación de niños/a y jóvenes hospitalizados para continuar con su proceso de enseñanza-aprendizaje, contribuir a 
una estabilidad emocional y un bienestar y calidad de vida (Polaino y Lizasoain, 1992). Así, aula hospitalaria es un escenario que permite a personas con enfermedades continuar realizando actividades socioeducativas (Lizasoain, 2007). En suma, la PH potencia una atención integral que implica interdisciplinariedad, por ello, es necesario forjar un marco común que no solo genere un código deontológico, sino que fomente un ethos interprofesional en $\mathrm{PH}$.

Ante este marco, los objetivos del presente artículo son:

a) Analizar y cartografiar los vínculos entre la Ética, la TE y la PH en experiencias educativas.

b) Proponer una dimensión transversal que fomente un ethos interprofesional, a través del uso de la TE, en el ámbito de la PH.

\section{MÉTODO}

Se realizó una revisión sistematizada de la literatura enfocada a las experiencias educativas que promueven vínculos entre la ética, la TE y la PH. La revisión sistematizada es una metodología que promueve transparencia y rigor (Hart, 2008) y permite identificar tendencias y oportunidades en futuras líneas de investigación (Manchado et al., 2009). La revisión sistematizada siguió el modelo propuesto por la Declaración PRISMA (Urrútia y Bonfill, 2010) y las fases de búsqueda, evaluación, análisis y síntesis de Codina (2018). Las directrices son:

\section{Criterios de inclusión}

- Periodo: del 2016 al 2021 que permita analizar estudios recientes.

- Idioma: Inglés y Español.

- Acceso: Open Access desde una cuenta universitaria en el Servicio de Biblioteca de la Universidad de las Islas Baleares.

- Tipo de documentos incluidos: limitados a artículos. Se respetó cualquier tipo de estudio.

- Áreas de investigación: En Scopus las subáreas fueron: Social Sciences, Arts and Humanities, Psychology, Multidisciplinary. En Web of Science las áreas de investigación fueron: Educational Research, Psychology, Science Technology Other Topics, Sociology, Philosophy.

\section{Identificación de las bases de datos}

- Las bases de datos fueron: Scopus, Web of Science, ERIC y Dialnet Plus.

- La última fecha de búsqueda fue el 24 de marzo de 2021.

\section{Establecimiento de la estrategia de búsqueda}

Se utilizó el buscador de tesauros de ERIC para identificar los conceptos centrales o descriptores. La Tabla 1 muestra la relación entre los términos y los descriptores. 
Tabla 1.

Descriptores y términos

\begin{tabular}{|c|c|c|c|}
\hline Descriptor ERIC & Término español & Término inglés & Vocablos cercanos \\
\hline ethics & Ética & Ethics & Moral \\
\hline Technology Education & Tecnología Educativa & Technology Education & $\begin{array}{c}\text { TIC } \\
\text { Educación tecnológica } \\
\text { Education/ Technology }\end{array}$ \\
\hline Pedagogy & Pedagogía Hospitalaria & Hospital Pedagogy & $\begin{array}{c}\text { Aula hospitalaria } \\
\text { Hospital School }\end{array}$ \\
\hline Inclusion & Inclusión educativa & Inclusive Education & Escuela inclusiva \\
\hline
\end{tabular}

Fuente: Elaboración propia.

Un segundo paso fue establecer la combinación de palabras clave para realizar ecuaciones de búsqueda (Tabla 2) con operadores booleanos y truncamientos adaptados a cada base de datos. Las ecuaciones de búsqueda responden a tres dimensiones: a) Relación Ética y TE; b) Relación Ética y PH; c) Relación TE y PH.

Tabla 2.

Proceso de búsqueda y cribado por criterios de inclusión

\begin{tabular}{|c|c|c|c|c|c|}
\hline $\begin{array}{l}\text { Base de } \\
\text { datos }\end{array}$ & $\begin{array}{l}\text { Ecuaciones de búsqueda según } \\
\text { dimensión }\end{array}$ & $\begin{array}{l}\text { Fecha de } \\
\text { búsqueda }\end{array}$ & $\begin{array}{l}\text { Primer } \\
\text { resultado }\end{array}$ & $\begin{array}{l}\text { Resultado } \\
\text { después de los } \\
\text { criterios de } \\
\text { inclusión }\end{array}$ & $\begin{array}{l}\text { Total } \\
\text { documentos } \\
\text { incluidos }\end{array}$ \\
\hline \multirow{3}{*}{$\begin{array}{l}\text { Web of } \\
\text { Science }\end{array}$} & $\begin{array}{l}\text { a. (ethics OR moral) AND ("technology } \\
\text { education" OR tic* OR "Digital } \\
\text { Competence") }\end{array}$ & \multirow{3}{*}{$24 / 03 / 2021$} & \multirow{3}{*}{$\begin{array}{l}945 \\
26\end{array}$} & \multirow{3}{*}{45} & \multirow{3}{*}{98} \\
\hline & $\begin{array}{l}\text { b. (ethics OR moral) AND (“Hospital } \\
\text { Pedagogy" OR “Educational Inclusion" } \\
\text { OR "student diversity") }\end{array}$ & & & & \\
\hline & $\begin{array}{l}\text { c. ("Digital Competence" OR } \\
\text { "Technology Education") AND } \\
\text { ("Hospital Pedagogy" OR Hospital* OR } \\
\text { Inclusion OR "student diversity") }\end{array}$ & & & & \\
\hline \multirow{3}{*}{ Scopus } & $\begin{array}{l}\text { a. (ethics OR moral) AND ("technology } \\
\text { education" OR tic* OR "Digital } \\
\text { Competence") }\end{array}$ & \multirow{3}{*}{$19 / 03 / 2021$} & \multirow{3}{*}{$\begin{array}{l}463 \\
20\end{array}$} & \multirow{3}{*}{25} & \multirow{3}{*}{57} \\
\hline & $\begin{array}{l}\text { b. (ethics OR moral) AND ( "Hospital } \\
\text { Pedagogy" OR "Educational } \\
\text { Inclusion" OR "student diversity" ) }\end{array}$ & & & & \\
\hline & $\begin{array}{l}\text { c. ("Digital Competence" OR } \\
\text { "Technology Education") AND } \\
\text { ("Hospital Pedagogy" OR Hospital* OR } \\
\text { Inclusion OR "student diversity") }\end{array}$ & & & & \\
\hline
\end{tabular}




\begin{tabular}{|c|c|c|c|c|c|}
\hline $\begin{array}{l}\text { Base de } \\
\text { datos }\end{array}$ & $\begin{array}{l}\text { Ecuaciones de búsqueda según } \\
\text { dimensión }\end{array}$ & $\begin{array}{l}\text { Fecha de } \\
\text { búsqueda }\end{array}$ & $\begin{array}{l}\text { Primer } \\
\text { resultado }\end{array}$ & $\begin{array}{l}\text { Resultado } \\
\text { después de los } \\
\text { criterios de } \\
\text { inclusión }\end{array}$ & $\begin{array}{l}\text { Total } \\
\text { documentos } \\
\text { incluidos }\end{array}$ \\
\hline \multirow{3}{*}{ ERIC } & $\begin{array}{l}\text { a. (ethics) AND ("Technology } \\
\text { Education" OR TIC* OR “Digital } \\
\text { competence") }\end{array}$ & \multirow{3}{*}{$12 / 03 / 2021$} & \multirow{3}{*}{490} & 23 & \multirow{3}{*}{138} \\
\hline & b. (ethics OR moral) AND hospital & & & 6 & \\
\hline & $\begin{array}{l}\text { c. ("Digital Competence" OR } \\
\text { "Technology Education") AND } \\
\text { ("Hospital Pedagogy" OR Hospital* OR } \\
\text { Inclusion OR "student diversity") }\end{array}$ & & & 109 & \\
\hline \multirow{3}{*}{$\begin{array}{l}\text { Dialnet } \\
\text { Plus }\end{array}$} & $\begin{array}{l}\text { a. (ética OR moral) AND (“tecnología } \\
\text { educativa" OR “competencia digital” } \\
\text { OR TIC* OR “Educación tecnológica”) }\end{array}$ & \multirow{3}{*}{$15 / 03 / 2021$} & \multirow{3}{*}{$\begin{array}{r}680 \\
1811\end{array}$} & \multirow{3}{*}{$\begin{array}{l}189 \\
22\end{array}$} & \multirow{3}{*}{331} \\
\hline & $\begin{array}{l}\text { b. (ética OR moral) AND ("pedagogía } \\
\text { hospitalaria" OR “aula hospitalaria" OR } \\
\text { hospital*) }\end{array}$ & & & & \\
\hline & $\begin{array}{l}\text { c. ("competencia digital" OR } \\
\text { "Tecnología educativa") AND } \\
\text { ("Pedagogía Hospitalaria" OR "aula } \\
\text { hospitalaria" OR “inclusión educativa" } \\
\text { OR "escuela inclusiva" OR diversidad) }\end{array}$ & & & & \\
\hline
\end{tabular}

Fuente: Elaboración propia

En total 624 documentos fueron incluidos para la fase de evaluación. Todos los documentos se cargaron en el gestor bibliográfico de Mendeley. Además, se comprobaron las referencias a través del gestor web de Endnote.

\section{Selección y evaluación de los estudios}

En primer lugar, se revisaron los títulos y resúmenes de los artículos recuperados; ello permitió eliminar artículos duplicados y realizar una clasificación según los criterios de inclusión del artículo. En segundo lugar, se evaluaron los artículos siguiendo el criterio de exclusión: relación con los objetivos de estudio. La Figura 1 muestra el diagrama de flujo, adaptado de Urrútia y Bonfill (2010), de la fase de evaluación. 
Figura 1.

Diagrama de flujo de la fase de evaluación

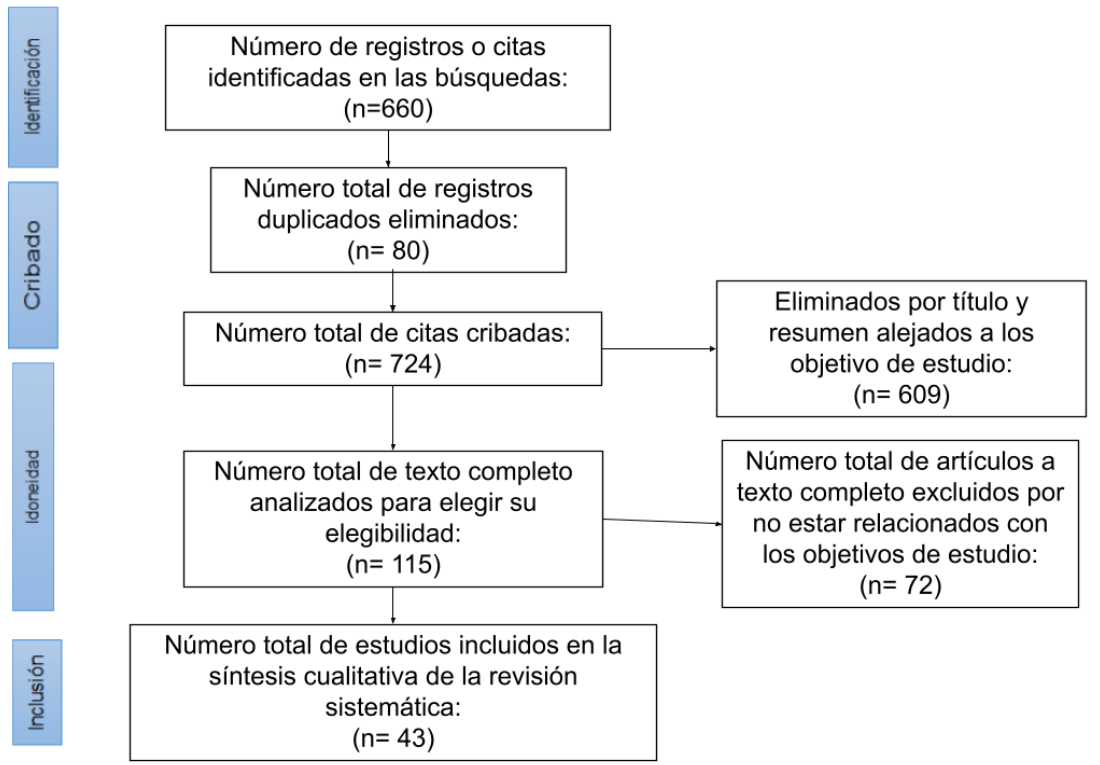

Nota: El resultado de la fase de evaluación fueron 43 artículos incluidos para la fase de análisis.

\section{Definición de las variables de estudio}

En la Tabla 3 se muestran las variables bibliométricas que determinan aspectos de los documentos que permiten el encuadre a un contexto determinado.

\section{Proceso de extracción de datos y confirmación de los mismos}

Se trabajó de manera conjunta en una carpeta compartida en el gestor bibliográfico de Mendeley. Se realizó una revisión por pares, en la cual, ambos autores revisaron los artículos y seleccionaron los resultados incluidos en la fase de síntesis. En supuesto de disconformidad, se dialogó en reuniones telemáticas.

\section{RESULTADOS}

Los resultados son presentados en dos fases. Primero, se presenta el análisis bibliométrico. Segundo, se sistematizan los artículos según su tipo de relación entre las tres dimensiones propuestas.

\subsection{Análisis bibliométrico}

La primera fase de análisis se compone por la división de variables bibliométricas que posibilitan una cartografía del contexto de los artículos seleccionados. En la Tabla 5 aparecen las variables bibliométricas de los artículos incluidos en la síntesis. 
Tabla 3.

Variables bibliométricas

\begin{tabular}{|c|c|c|c|c|c|}
\hline Base de datos & Autores & Año & $\begin{array}{c}\text { Tipo de } \\
\text { investigación }\end{array}$ & País & Idioma \\
\hline Dialnet Plus & $\begin{array}{l}\text { Ardón, D. } \\
\text { Leytón, F., } \\
\text { Méndez, N., } \\
\text { Monge, K., } \\
\text { Valverde, G. }\end{array}$ & 2017 & $\mathrm{CL}$ & Costa Rica & Español \\
\hline Scopus & $\begin{array}{l}\text { Baharuddin, B. } \\
\text { Delle, J.2 }\end{array}$ & 2019 & $\mathrm{IBD}$ & Indonesia & Inglés \\
\hline Dialnet Plus & Balladares, J. & 2017 & $\mathrm{IT}$ & Ecuador & Español \\
\hline Dialnet Plus & $\begin{array}{l}\text { Bonilla-del-rio, M, García-Ruíz, R., } \\
\text { Pérez Rodríguez M. A.r2 }\end{array}$ & 2018 & $\mathrm{CL}$ & España & Español \\
\hline ERIC & $\begin{array}{l}\text { Bublic, M.1, } \\
\text { Blatnik, S.2, } \\
\text { Eminovic, F.3, } \\
\text { Selimovic, S.2 } \\
\text { Šaric, E. } 4\end{array}$ & 2018 & $\mathrm{CT}$ & $\begin{array}{l}1 \text { Croacia, } \\
2 \text { Eslovenia } \\
3 \text { Serbia } \\
4 \text { Bosnia y } \\
\text { Herzegovina }\end{array}$ & Inglés \\
\hline Scopus & $\begin{array}{l}\text { Cámara, A. } \\
\text { Díaz, E. M. } \\
\text { Ortega-Tudela, J.M. }\end{array}$ & 2017 & $\mathrm{CL}$ & España & Español \\
\hline Dialnet Plus & $\begin{array}{l}\text { Contreras, S. } \\
\text { Romero, F. }\end{array}$ & 2020 & $\mathrm{CL}$ & Chile & Español \\
\hline $\begin{array}{l}\text { Web of } \\
\text { Science }\end{array}$ & $\begin{array}{l}\text { Crescenzi-Lanna, L. } \\
\text { Valente, R. } \\
\text { Suárez-Gómez, R. } \\
\end{array}$ & 2019 & AC. & España & Español \\
\hline Scopus & $\begin{array}{l}\text { Crespo, F. } \\
\text { Sánchez, C. }\end{array}$ & 2019 & $\mathrm{IM}$ & España & Español \\
\hline ERIC & $\begin{array}{l}\text { Dementievich, } \mathrm{P} \text {. } \\
\text { Yurievna, } \mathrm{O}\end{array}$ & 2017 & IES & Rusia & Inglés \\
\hline ERIC & Duncan, L. & 2020 & IT & USA & Inglés \\
\hline Scopus & $\begin{array}{l}\text { Fierros-Sanchez-Cuenca, M. } \\
\text { Bengoechea-Menéndez, C. } \\
\text { Yáñez-Cañas, S. } \\
\text { Martinez-Naranjo, C. } \\
\text { López-Ibor, B. }\end{array}$ & 2020 & $\mathrm{CT}$ & España & Español \\
\hline $\begin{array}{l}\text { Web of } \\
\text { Science }\end{array}$ & $\begin{array}{l}\text { Flujas, J.M., } \\
\text { Ruiz-Castañeda, D., } \\
\text { Botella Arbona, C. } \\
\text { Gómez Becerra, I } \\
\end{array}$ & 2017 & $\mathrm{CL}$ & España & Español \\
\hline Scopus & $\begin{array}{l}\text { Frolova, T. N. } \\
\text { Umarova, Z. Y. } \\
\text { Suhorukhih, A. V. } \\
\text { Lazareva, Y. B. } \\
\end{array}$ & 2020 & $\mathrm{CT}$. & Rusia & Inglés \\
\hline Dialnet Plus & García, A. & 2017 & $\mathrm{IT}$ & España & Español \\
\hline $\begin{array}{l}\text { Web of } \\
\text { Science }\end{array}$ & García, M. & 2020 & IT. & España & Español \\
\hline ERIC & Ghazi, M. & 2018 & $\mathrm{CL}$ & Arabia Saudi & Inglés \\
\hline Dialnet Plus & Gil, M.G. & 2021 & $\mathrm{IT}$ & Colombia & Español \\
\hline
\end{tabular}




\begin{tabular}{|c|c|c|c|c|c|}
\hline Base de datos & Autores & Año & $\begin{array}{c}\text { Tipo de } \\
\text { investigación }\end{array}$ & País & Idioma \\
\hline ERIC & $\begin{array}{l}\text { Kim, J. } \\
\text { Kimm, C. H. }\end{array}$ & 2017 & $\mathrm{RL}$ & USA & Inglés \\
\hline ERIC & $\begin{array}{l}\text { Korsgaard, E. } \\
\text { Voldborg, H. }\end{array}$ & 2017 & $\mathrm{CL}$ & Dinamarca & Inglés \\
\hline $\begin{array}{l}\text { Web of } \\
\text { Science }\end{array}$ & $\begin{array}{l}\text { López-Meneses, E. } \\
\text { Fernández-Cerero, J. }\end{array}$ & 2020 & $\mathrm{CL}$ & España & Español \\
\hline Dialnet Plus & Martínez, E. & 2019 & $\mathrm{IT}$ & España & Español \\
\hline Dialnet Plus & $\begin{array}{l}\text { Mauri, E. } \\
\text { Carrera, X. } \\
\text { Selga, M, } \\
\text { López, C. } \\
\text { Macià, M. }\end{array}$ & 2016 & $\mathrm{CL}$ & España & Español \\
\hline Scopus & $\begin{array}{l}\text { Mombaers, T. } \\
\text { Donche, } \mathrm{V} .\end{array}$ & 2020 & $\mathrm{CL}$ & Bélgica & Inglés \\
\hline ERIC & $\begin{array}{l}\text { Montenegro, M. } \\
\text { Fernández, J. }\end{array}$ & 2019 & $\mathrm{CL}$ & España & Inglés \\
\hline Dialnet Plus & $\begin{array}{l}\text { Negre, F. } \\
\text { Verger, } \mathrm{S} \text {. }\end{array}$ & 2017 & IT & España & Español \\
\hline $\begin{array}{l}\text { Web of } \\
\text { Science }\end{array}$ & $\begin{array}{l}\text { Novella-Garcia, C. Cloquell-Lozano, } \\
\text { A. }\end{array}$ & 2021 & $A C$ & España & Inglés \\
\hline $\begin{array}{l}\text { Web of } \\
\text { Science }\end{array}$ & $\begin{array}{l}\text { Ochoa-Aizpurua, B. } \\
\text { Correa, J. M., } \\
\text { Gutiérrez-Cabello, A. }\end{array}$ & 2019 & $\mathrm{CL}$ & España & Español \\
\hline Dialnet Plus & $\begin{array}{l}\text { Palmdorf, S, } \\
\text { Nadolny, S. } \\
\text { Hochmuth, A., } \\
\text { Lea Stark, A. } \\
\text { Dockweiler, C. }\end{array}$ & 2019 & IT & Alemania & Inglés \\
\hline Dialnet Plus & $\begin{array}{l}\text { Palomares-Ruiz, A., Sánchez- } \\
\text { Navalón, B., } \\
\text { Garrote-Rojas, D. }\end{array}$ & 2016 & IM & España & Español \\
\hline $\begin{array}{l}\text { Web of } \\
\text { Science }\end{array}$ & $\begin{array}{l}\text { Pellicano, L. } \\
\text { Bölte, } \mathrm{S} \text {. } \\
\text { Stahmer, A. }\end{array}$ & 2018 & $\mathrm{IT}$ & $\begin{array}{l}1 \text { Australia } \\
2 \text { Suecia } \\
3 \text { USA } \\
\end{array}$ & Inglés \\
\hline Dialnet Plus & $\begin{array}{l}\text { Pereiro, M. del C. } \\
\text { Penas, Y. }\end{array}$ & 2017 & $\mathrm{IBD}$ & España & Español \\
\hline Scopus & $\begin{array}{l}\text { Peterson-Ahmad, M. B. } \\
\text { Stepp, J. B. } \\
\text { Somerville, K. }\end{array}$ & 2018 & $\mathrm{IM}$ & USA & Inglés \\
\hline ERIC & $\begin{array}{l}\text { Roberts-Yates, C., } \\
\text { Silvera-Tawil, D. }\end{array}$ & 2019 & $\mathrm{CL}$ & Australia & Inglés \\
\hline Scopus & $\begin{array}{l}\text { Saladino, M., } \\
\text { Martin, D., } \\
\text { San Martín, Á. } \\
\end{array}$ & 2020 & $\mathrm{CL}$ & España & Español \\
\hline Dialnet Plus & Salgado, C. & 2020 & $\mathrm{IT}$ & Colombia & Español \\
\hline Scopus & $\begin{array}{l}\text { Sani-Bozkurt, S. } \\
\text { Vuran, S., } \\
\text { Akbulut, Y. }\end{array}$ & 2017 & $\mathrm{IBD}$ & Turquía & Inglés \\
\hline Dialnet Plus & $\begin{array}{l}\text { Serrano, J. L. } \\
\text { Castañeda, L. J }\end{array}$ & 2016 & IM & España & Español \\
\hline
\end{tabular}




\begin{tabular}{|c|c|c|c|c|c|}
\hline Base de datos & Autores & Año & $\begin{array}{c}\text { Tipo de } \\
\text { investigación }\end{array}$ & País & Idioma \\
\hline $\begin{array}{l}\text { Web of } \\
\text { Science }\end{array}$ & $\begin{array}{l}\text { Solovieva, O. V. } \\
\text { Palieva, N. A. } \\
\text { Borozinets, N. M. } \\
\text { Kozlovskaya, G. Y. } \\
\text { Prilepko, J. V. }\end{array}$ & 2020 & $\mathrm{CT}$ & Rusia & Inglés \\
\hline ERIC & $\begin{array}{l}\text { Thomas, J. } \\
\text { Brevetti, M }\end{array}$ & 2017 & IT & USA & Inglés \\
\hline Dialnet Plus & $\begin{array}{l}\text { Tumino, M. C. } \\
\text { Bournissen, J. M }\end{array}$ & 2017 & $\mathrm{IM}$ & Argentina & Español \\
\hline Dialnet Plus & Vico, $\mathrm{P}$ & 2019 & $\mathrm{CL}$ & España & Español \\
\hline Scopus & $\begin{array}{l}\text { Yáñez, S. } \\
\text { Fierros, M., Bengoechea- } \\
\text { Menéndez, C. } \\
\text { López-Ibor, B. }\end{array}$ & 2020 & $\mathrm{CL}$ & España & Español \\
\hline
\end{tabular}

Nota: AC-Análisis de contenido; CL-Cualitativa; CT-Cuantitativa; IBD-Investigación Basada en Diseño; IESInvestigación Estratégica Sistemática; IM-Investigación Mixta; IT-Investigación Teórica; RL-Revisión de la Literatura

En la variable de base de datos, se destacan 16 artículos de Dialnet Plus, 11 de Scopus, 9 de Eric y 7 de Web of Science. Por otro lado, en la Figura 2 se aprecia cómo en 2017 fue el año con un mayor número de artículo y como en 2020 hubo un repunte con 11 artículos. En cuanto a la publicación por país, España tiene 21 registros, seguida de USA (5), Rusia (3), Australia (2), Colombia (2) y los demás países con 1 registro. En cuanto al idioma, el 58\% son artículos en Español y el $42 \%$ en Inglés.

Figura 2.

Evolución de artículos por año

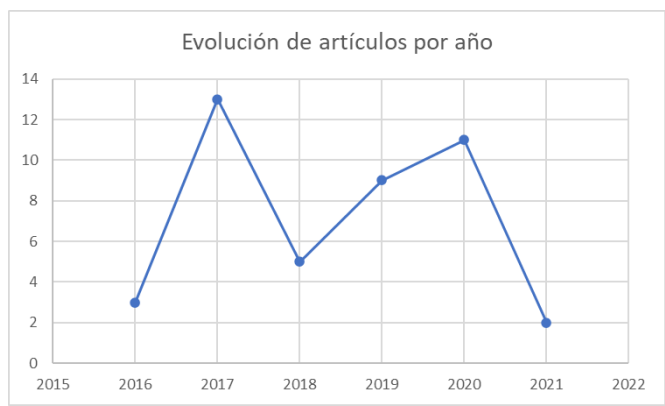

\subsection{Sistematización según dimensiones}

Se presentan los resultados siguiendo las tres dimensiones de relación. En la intersección de las tres dimensiones se pretenden encontrar ejes transversales que posibiliten una cuarta dimensión con compromisos éticos, a través del uso de la TE, en el ámbito de la PH. Esta cuarta dimensión surgió tras la fase de análisis y síntesis de los resultados. Los estudios fueron divididos por su filiación a alguna de las cuatro dimensiones de relación.

\subsubsection{Relación entre Ética y Tecnología Educativa}

En cuanto al profesorado, Novella-García y Cloquell-Lozano (2021) analizaron diferentes planes de estudio (118) de los Grados de Educación Infantil y Educación Primaria en España en donde 
se trabaje la competencia digital desde una dimensión ética. Sus resultados muestran que la dimensión ética solo fue incorporada en el $26 \%$ de los planes de formación en competencia digital. Por otro lado, los decentes identifican las TIC como instrumentos promotores de valores (Tumino y Bournissen, 2017) Por último, Frolova et al. (2020) presentan un análisis de la alfabetización socio-ética en TIC de profesores de universidades rusas. Los resultados muestran que los docentes poseen un elevado nivel sobre alfabetización ética, sin embargo, no comprenden la funcionalidad de promover estas habilidades para el buen uso tecnológico.

En cuanto al alumnado, ante el nuevo perfil de discentes del siglo XXI es necesario considerar el desarrollo de unos valores que guíen el uso ético de las TIC (Balladares, 2017). En García Rodríguez (2020) se fomente una cultura científica crítica y responsable para llevar a cabo en las aulas basada en una educación Steam\&Ethics.

\subsubsection{Relación entre Ética y Pedagogía Hospitalaria}

Aparecen dos relaciones que pueden complementarse: la relación entre la ética y la PH y la relación entre la ética y la inclusión educativa.

En cuanto al profesorado, es vital definir el perfil de profesorado (Ardón et al., 2017), siendo la afectividad una característica esencial para el fomento de inclusión, incremento de situaciones dialógicas y el acompañamiento (Contreras y Romero, 2020). Por otro lado, Pellicano (2018) critica que, tras un estudio utilizando el inventario INCLUSIO (herramienta de evaluación de la inclusión escolar), existe escepticismo y escasa aplicación de la inclusión educativa en las aulas. En suma, ante la crisis de valores existentes en la sociedad actual, se precisan cambios en la educación para favorecer habilidades reflexivas como autonomía, autoconocimiento, superación y empatía (Gil, 2021).

En relación al alumnado, se describen estudios sobre la motivación favorece el seguimiento académico en estudiantes hospitalizados (Fierros-Sanchez-Cuenca et al., 2020; Mombaers y Donche, 2020). Los resultados legitiman que la motivación del alumnado se vea perjudicada durante los procesos de enfermedad, aunque esta disminución en su motivación académica puede ser temporal. Para mejorar la atención a estos sujetos es necesario: incremento de recursos múltiples y de calidad, continuidad en la formación docente y aumento de la concienciación (Crespo y Sánchez, 2019). Por último, Duncan (2020) explora el papel de la educación en la vida y el trabajo de Blanche Van Leuven Browne en la escuela-hospital de Detroit para niños físicamente discapacitados; se muestra la necesidad de cambio, los discentes deben convivir en ambientes igualitarios donde se favorezcan las oportunidades éticas e inclusivas.

\subsubsection{Relación entre Tecnología Educativa y Pedagogía Hospitalaria}

El uso de las TIC favorece la inclusión de alumnado con algún déficit de atención en las escuelas (Korsgaard y Voldborg, 2017); además, el uso de las TIC no debe quedar anclado en las infraestructuras o uso de aparatos, ante esto, se debe avanzar e invertir en formación y en recursos humanos para ampliar las posibilidades del uso de las TIC en la atención a la diversidad (Ochoa-Aizpurua et al., 2019) y fomentar modelos dirigidos a la inteligencia digital (Solovieva et al., 2020). 
En referencia a los docentes, se destaca la posibilidad de cambio hacia entornos digitales inclusivos, siendo los docentes los agentes personales que analizan las necesidades y ayudan a poner en práctica estrategias y adaptaciones en el aula ordinaria (Bublic et al., 2018; Ghazi Abed, 2018). Las tecnologías, debido a su naturaleza motivadora y las posibilidades que ofrecen a nivel de adaptación de acuerdo con las características individuales y los ritmos de aprendizaje de cada una, favorecen los procesos de inclusión (Saladino et al., 2020). Por tanto, es menester poner en primer orden la formación continua del profesorado, incluidos materiales y estrategias de adaptación tecnológica, sobre el uso correcto de las TIC en alumnado con alguna diversidad funcional (López y Fernández, 2020).

Según las perspectivas del profesorado, las principales barreras en el desarrollo de planes docentes con el uso de las TIC para personas con alguna discapacidad, son: "falta de tiempo", la "actitud del profesorado", factores "económicos", escasa oferta de formación, escasos estudiantes con alguna capacidad diferente, distancias geográficas, calidad de la formación y edad del profesorado (Montenegro y Fernández, 2019).

Respecto al alumnado, existen múltiples oportunidades para adaptar la tecnología Web 2.0, la educomunicación y la integración de robots sociales para que sea adecuada para los estudiantes en contextos específicos de educación especial (Bonilla-del-rio et al., 2018; Peterson-Ahmad et al., 2018; Roberts-Yates y Silvera-Tawil, 2019).

Por otra parte, diversos proyectos fomentan la competencia digital educativa en ámbitos de inclusión, como la creación de un prototipo de E-learning para profesores que muestra notables beneficios en estudiantes con necesidades especiales (Baharuddin y Dalle, 2019), la introducción del pensamiento computacional a través de una plataforma SCRATH (Pereiro y Penas, 2017), el diseño de historias sociales interactivas en niños con Trastorno del Espectro Autista.(Sani-Bozkurt et al., 2017) y un programa para paliar el dolor y la ansiedad en pacientes pediátricos a través de la realidad virtual y una plataforma de tele-psicología (Flujas et al., 2017)

En cuanto al uso de aplicaciones, suscitan al profesorado a considerar características de los recursos educativos digitales: motivación, accesibilidad, mecanismos de seguridad; exposición y acceso a contenidos inapropiados; integración de herramientas de adaptación; adecuación del contenido a la edad; y el componente verbal de la app. (Crescenzi-Lanna et al., 2019; Mauri Maldonado et al., 2016). El proyecto EDUMOBSPITALARIOS creó un metacatálogo de Apps para uso educativo y un catálogo de actividades didácticas con tecnologías móviles, especialmente diseñadas para el contexto de aulas hospitalarias (Serrano y Castañeda, 2016). En cuanto al uso de dispositivos móviles hay notables beneficios en su uso en personas con Discapacidades Intelectuales (Kim y Kimm, 2017) Por otro lado, Vico (2019) analiza el uso de la Pizarra Digital Interactiva (PDI) en el proceso de enseñanza-aprendizaje con alumnos con NEE. Los resultados indican que no a todo el alumnado con NEE satisface sus necesidades educativas prioritarias, además, pueden producción falta de interacción y reciprocidad.

Por último, dos proyectos fomentan propuestas innovadoras con metodología AprendizajeServicio en un ámbito universitario (Cámara et al., 2017; Negre y Verger, 2017). Además, el proyecto de $\mathrm{PH}$ en la Unidad de Trastornos del Comportamiento Alimentario (UTCA) del Hospital Universitario Nuestra Señora del Perpetuo Socorro, de Albacete, España destaca la perspectiva positiva del alumnado por superar su curso académico con éxito, la enseñanza 
personalizada de los docentes del Aula Hospitalaria, y la posibilidad de evadirse del medio hospitalario.(Palomares-Ruiz et al., 2016)

\subsubsection{Experiencias de vínculos entre compromisos éticos, a través de la Tecnología Educativa, en el ámbito de la Pedagogía Hospitalaria}

Aunque una primera búsqueda no haya arrojado estudios sobre vínculos entre ética, TE y PH, durante el proceso de análisis se encontraron tres propuestas que trazan ejes transversales. García Fernández (2017) analiza el marco teórico relacionado con los principios éticos de discentes hospitalizados. La ética docente favorece la atención integral e individualizada del estudiante. En relación con los principios morales son esenciales: la justicia, la igualdad y la responsabilidad de los profesionales. En Martínez-Navarro (2019), tras un análisis teórico, surge una nueva fórmula de imperativo ético en el ámbito de la inclusión educativa: "tratemos a todas las personas de modo que no sean dañadas y al mismo tiempo sean beneficiadas en cuanto a reforzar sus capacidades, en cuanto a aumentar su empoderamiento" (2019, p. 5). El empoderamiento podrá surgir a partir de las tecnologías asistenciales. Palmdorf et al. (2019) analizan los dilemas y las oportunidades éticas de la atención a Personas con Demencia (PcD) en el uso de tecnologías asistenciales. La integración de escenarios técnicos sobre el futuro del cuidado dentro del concepto de Planificación Anticipada de la Atención ofrece la posibilidad de abordar los problemas éticos de forma prospectiva. En suma, desde esta nueva vertiente, el propósito de un aula hospitalaria no debe ser solo académico, pues tiene un papel fundamental en el objetivo de ser herramienta que posibilite formación biopsicosocial y espiritual. (Cañas et al., 2020). Las aulas hospitalarias brindan un escenario de crecimiento personal y de apoyo educativo y biopsicosocial a niñas, niños, adolescentes y jóvenes (Salgado, 2020).

\section{DISCUSIÓN Y CONCLUSIONES}

La revisión sistematizada propició cuatro vías de interrelación entre la Ética, la TE y la PH. Bajo estos cuatro vínculos es posible construir un carácter moral interdisciplinar, enriquecido por la $T E$, lo cual legitima la novedad de la revisión sistematizada realizada.

a) Relación entre Ética y TE. Los resultados permiten identificar, de manera descriptiva, estudios sobre nuevos perfiles morales, tanto de profesorado como de alumnado, relacionados con diversos valores en un entorno digital educativo (Balladares, 2017; García, 2020; Tumino y Bournissen, 2017).

b) Relación entre Ética y PH. Entre las perspectivas se resalta la motivación académica del alumnado en respuesta a programas en PH o en inclusión educativa (Fierros-Sanchez-Cuenca et al., 2020; Mombaers y Donche, 2020). Aunque haya buenas prácticas de inclusión, el estudio de Pellicano (2018) critica el escaso de compromiso por aplicar la inclusión educativa en aulas de Suecia.

c) Relación entre TE y PH. Se hace patente que hay un notable desarrollo entre el uso de la TE en entornos inclusivos o en $\mathrm{PH}$. Aunque los artículos seleccionados en esta dimensión no mencionaran de manera patente una perspectiva ética, toda acción humana tiene un talente moral que se refleja en buenas prácticas educativas (Domingo, 2008). Por tanto, esta dimensión 
da cabida a indicios latentes de la relación entre compromisos docentes o buenas prácticas, a través del uso de la TE, en el ámbito de la inclusión educativa y la PH.

d) Experiencias de vínculos entre compromisos éticos, a través de la Tecnología Educativa, en el ámbito de la PH. En esta cuarta dimensión se sistematizaron artículos que manifestaran una relación directa entre las tres dimensiones de estudio. Aunque son pocos artículos los que aparecen en esta dimensión, hay indicios de vínculos y proximidad entre los ámbitos de estudio.

Además, la presente revisión atañe un ámbito poco indagado. Solo se han encontrado dos estudios que mantienen cierta relación con el presente trabajo, sin embargo, ninguno relaciona la ética, la TE y la PH. En García (2019) se realiza una revisión sobre la resiliencia y su relación con la Pedagogía Hospitalaria. En Vaquero et.al. (2016) se analizan los beneficios de las TIC en planes sociofamiliares. Por tal, la revisión sugiere una oportunidad para construir una línea futura de investigación desde la cual, la perspectiva moral cimiente buenas prácticas con TE dentro de la PH. Ahora bien, los resultados deben tomarse con cautela.

En consonancia con lo anterior, una de las limitaciones de la revisión ha sido los escasos resultados en las búsquedas, si se unen términos de los tres ejes del estudio. Un ejemplo de ello es utilizando la ecuación de búsqueda (ética OR moral) AND ("tecnología educativa" OR TIC OR "competencia digital") AND ("pedagogía hospitalaria" OR "aula hospitalaria") en Dialnet Plus dará cero resultados. En Scopus y WOS tampoco hay resultados con la búsqueda: (ethics OR moral) AND ("Technology Education" OR tic OR "Educational Technology") AND ("Hospital Pedagogy" OR "Hospital Classroom"). Sin embargo, ante la dificultad se estableció un silogismo dado por las diferentes relaciones mencionadas que permitiera indagar posibles nexos entre estos tres ejes.

Como cierre, deseamos que este trabajo sirva como impulso para mejorar e incrementar la investigación, de manera rigurosa, referente a qué estrategias pueden fomentar compromisos éticos y buenas prácticas, utilizando el potencial y la constante innovación en TE para mejorar la inclusión educativa del alumnado y sus familias dentro del ámbito de la PH. Así, los retos enunciados por el informe de la UNESCO (2015) sobre la necesidad de una educación inclusiva y desde una perspectiva humanista y holística también son suscritos por la PH. En este sentido, la inclusión educativa se vuelve un reto primordial para el desarrollo de buenas prácticas educativas (Molina y Verger, 2020).

\section{REFERENCIAS}

Ainscow, M. (2019). 25 años después de la Declaración de Salamanca de la UNESCO Crear sistemas educativos inclusivos y equitativos. Documento de Discusión Preparado Para El Foro Internacional Sobre Inclusión y Equidad., 11-13.

Ardón Esquivel, D., Leytón Vega, F., Méndez Rodríguez, N., Monge Brenes, K. y Valverde Cabezas, G. (2017). La pedagogía hospitalaria en Costa Rica: La atención a la niñez menor de siete años de edad. Actualidades Investigativas En Educación, 17(1). https://doi.org/10.15517/aie.v17i1.27295 
Baharuddin, B. y Dalle, J. (2019). Transforming Learning Spaces for Elementary School Children with Special Needs. Journal of Social Studies Education Research, 10(2), 344-365.

Balladares, J. (2017). Una ética digital para las nuevas generaciones digitales. Revista PUCE, 104, 543-563. https://doi.org/10.26807/revpuce.v0i0.81

Bonilla-del-rio, M., García-Ruíz, R., \& Pérez Rodríguez, M. A. . (2018). La educomunicación como reto para la educación inclusiva. EDMETIC, Revista de Educación Mediática y TIC, 7(1), 66-86. https://doi.org/10.21071/edmetic.v7i1.10029

Bublic, M., Blatnik, S., Eminovic, F., Selimovic, S. y Šaric, E. (2018). Information and Communication Technology in Education and Rehabilitation of Children with Developmental Disabilities. 9th International Congress on New Trends in Education - ICONTE, 8(2), 5-7.

Cámara Estrella, Á. M., Díaz Pareja, E. M. y Ortega-Tudela, J. M. (2017). Aprendizaje-Servicio en la Universidad: Ayudando a la escuela a atender a la diversidad a través de las TIC. Bordón, 69(3), 73-87. https://doi.org/10.13042/Bordon.2017.51320

Cañas, S. Y., Sánchez-Cuenca, M. F., Menéndez, C. B. y Aliño, B. L. I. (2020). Healing a student, not just his or her disease. A case description. Psicooncologia, 17(2), 375-385. https://doi.org/10.5209/psic.72023

Carretero, S., Vuorikari, R. y Punie, Y. (2017). DigComp 2.1: The Digital Competence Framework for Citizens. With eight proficiency levels and examples of use. In EUR 28558 EN. Publications Office of the European Union.

Carta Europea de los Derechos del niño hospitalizado. (1986).

Codina, L. (2018). Revisiones bibliográficas sistematizadas. Procedimientos generales y Framework para Ciencias Humanas y Sociales. Máster Universitario en Comunicación Social. Departamento de Comunicación. Universitat Pompeu Fabra [documento en pdf, acceso: eRepositorio UPF ].

Contreras Salinas, S. y Romero Ormeño, F. (2020). Concepciones sobre afectividad en docentes que trabajan en aulas hospitalarias en Chile. Educación, 29(56), 27-47. https://doi.org/10.18800/educacion.202001.002

Crescenzi-Lanna, L., Valente, R. y Suárez-Gómez, R. (2019). Aplicaciones educativas, seguras e inclusivas: La protección digital desde una perspectiva ética y crítica. Comunicar, 27(61). https://doi.org/10.3916/c61-2019-08

Crespo Molero, F. y Sánchez Romero, C. (2019). Alumnado con Trastorno Mental Grave: análisis de la atención educativa recibida en la Comunidad de Madrid. Psychology, Society, \& Education, 11(1), 113. https://doi.org/10.25115/psye.v11i1.2124

Domingo Moratalla, A. (2008). Ética para educadores. PPC.

Duncan, L. (2020). 'Every One of Them Are Worth It': Blanche Van Leuven Browne and the Education of the 'Crippled Child'. History of Education Quarterly, 60(3), 324-350. https://doi.org/10.1017/heq.2020.28 
Esteban Bara, F. (2018). Ética del profesorado. Herder Editorial.

Ferrari, A. (2012). Digital Competence in Practice: An Analysis of Frameworks. JRC-IPTS.

Fierros-Sanchez-Cuenca, M., Bengoechea-Menendez, C., Yanez-Canas, S., Martinez-Naranjo, C. y Alino, B. L. I. (2020). A school in a paediatric oncology unit: The crucial role of education in the context of a serious illness. Revista Electronica Educare, 24(1). https://doi.org/10.15359/ree.24-1.5

Fierros-Sanchez-Cuenca, M., Bengoechea-Menéndez, C., Yáñez-Cañas, S., Martinez-Naranjo, C. y López-Ibor Aliño, B. (2020). Un colegio en una unidad de oncología pediátrica: El papel crucial de la educación en el contexto de una enfermedad grave. Revista Electrónica Educare, 24(1), 1-16. https://doi.org/10.15359/ree.24-1.5

Flujas Contreras, J. M., Ruiz-Castañeda, D., Botella, C. y Gómez, I. (2017). Un programa de bienestar emocional basado en Realidad Virtual y Terapia Online para enfermedades crónicas en infancia y adolescencia: La Academia Espacial. Revista de Psicología Clínica Con Niños y Adolescentes, 4(3), 17-25.

Frolova, T. N., Umarova, Z. Y., Suhorukhih, A. V. y Lazareva, Y. B. (2020). ICT enabled education: ethical and axiological competence formation. Opción: Revista de Ciencias Humanas y Sociales, 36(27), 322-340.

García-Gutiérrez, J. (2013). Aproximación ética a la competencia digital. Los niveles de uso y sentido en ámbitos educativos virtuales. Teoría de La Educación. Educación y Cultura En La Sociedad de La Información, 14(3), 121-145.

García Fernández, A. (2017). Fundamentos éticos para la práctica docente en el ámbito hospitalario. Dilemata, 9(23), 159-174.

García Parra, M. (2019). Ética y resiliencia familiar para una atención integral en el campo de las enfermedades raras. International Journal of Developmental and Educational Psychology, 1(2), 271-280. https://doi.org/10.17060/ijodaep.2019.n2.v1.1696

García Rodríguez, M. (2020). Los valores éticos y su relación con la ciencia y la tecnología. Una propuesta de trabajo en el aula. Haser. Revista Internacional de Filosofía Aplicada, 11, 125161. https://doi.org/10.12795/haser/2020.i11.05

Ghazi Abed, M. (2018). Teachers' Perspectives Surrounding ICT Use amongst SEN Students in the Mainstream Educational Setting. World Journal of Education, 8(1), 6-16. https://doi.org/10.5430/wje.v8n1p6

Gil Claros, M. G. (2021). Escuela, actitud ética y hospitalidad en la mirada del Otro. Revista de Educación y Pensamiento, 27(27), 56-59.

Gónzalez, J. (1996). El ethos, destino del hombre. UNAM-FCE.

González, M. J. M., Rivoir, A., Lázaro-Cantabrana, J. L. y Gisbert-Cervera, M. (2020). ¿Cuánto importa la competencia digital docente? Análisis de los programas de formación inicial docente en Uruguay. Innoeduca. International Journal of Technology and Educational Innovation, 6(2), 
128-240. https://doi.org/10.24310/innoeduca.2020.v6i2.5601

Grau Rubio, C. y Ortiz González, C. (2001). La pedagogía hospitalaria en el marco de una educación inclusiva. Ediciones Aljibe.

Hart, C. (2008). Doing a Literature Review: Releasing the Social Science Research Imagination. SAGE.

Kim, J. y Kimm, C. H. (2017). Functional Technology for Individuals with Intellectual Disabilities: Meta-Analysis of Mobile Device-Based Interventions. Journal of Special Education Apprenticeship, 6(1), 1-22.

Korsgaard Sorensen, E. y Voldborg Andersen, H. (2017). Strengthening Inclusion of Learners with Attention Difficulties through Interventions with Digital Technology in Processes of Production. European Journal of Open, Distance and E-Learning, 20(1), 45-60.

Lizasoain, O. (2007). Hacia un modo conjunto de entender la Pedagogía Hospitalaria. Primera Jornada Nacional de Pedagogía Hospitalaria En Venezuela, 1-15.

López Meneses, E. y Fernández Cerero, J. (2020). Tecnologías de la Información y la Comunicación y diversidad funcional. Conocimiento y formación del profesorado en Navarra. IJERI: International Journal of Educational Research and Innovation, 14, 59-75. https://doi.org/10.46661/ijeri.4407

Manchado Garabito, R., Tamames Gómez, S., López González, M., Mohedano Macías, L., D’Agostino, M. y Veiga de Cabo, J. (2009). Revisiones Sistemáticas Exploratorias. Medicina y Seguridad Del Trabajo, 55(216), 12-19. https://doi.org/10.4321/s0465-546×2009000300002

Martínez Navarro, E. (2019). Tecnologías asistenciales y dignidad de las personas. Dilemata, 30(30), $1-11$.

Mauri Maldonado, E., Carrera Farran, X., Selga Casarramona, M., Lopez Arias, C. y Macià Golobardes, M. (2016). Análisis de experiencias educativas con dispositivos móviles para una educación inclusiva. EDUTEC. Revista Electrónica de Tecnología Educativa, 56, 48-59. https://doi.org/10.21556/edutec.2016.56.658

Molina Garuz, M. C. y Verger Gelabert, S. (2020). Perspectivas y desafíos de la práctica profesional y la investigación en pedagogía hospitalaria. In T. Lleixà, Z. Bozu, \& A. Aneas (Eds.), Educación 2020-2022. Retos, tendencias y compromisos (pp. 47-52). IRE-UB.

Mombaers, T. y Donche, V. (2020). Hospital School Students' Academic Motivation and Support Needs: A Self-Determination Perspective. Frontiers in Education, 5. https://doi.org/10.3389/feduc.2020.00106

Montenegro Rueda, M. y Fernández Cerero, J. (2019). Main Barriers to ICT Teacher Training and Disability. Research in Social Sciences and Technology, 4(2), 96-114. https://doi.org/10.46303/ressat.04.02.7

Negre Bennasar, F. y Verger Gelabert, S. (2017). INEDITHOS: un proyecto de pedagogía hospitalaria dedicado a la mejora de la calidad de vida de niños y jóvenes con enfermedades raras a partir de la intervención e investigación con voluntariado universitario. Aula, 23(0), 107-119. 
https://doi.org/10.14201/aula201723107119

Novella-Garcia, C. y Cloquell-Lozano, A. (2021). The ethical dimension of digital competence in teacher training. Education and Information Technologies. https://doi.org/10.1007/s10639021-10436-z

Ochoa-Aizpurua Aguirre, B., Correa Gorospe, J. M. y Gutiérrez-Cabello Barragán, A. (2019). Las TIC en la atención a la diversidad educativa: el caso de la Comunidad Autónoma Vasca. Revista de Educación a Distancia (RED), 19(61), 1-21. https://doi.org/10.6018/red/61/07

Palmdorf, S., Nadolny, S., Hochmuth, A., Lea Stark, A. y Dockweiler, C. (2019). Ethical Challenges by Using Assistive Technologies in Dementia Home Care - Potential of Advance Care Planning. Dilemata, 30, 13-26.

Palomares-Ruiz, A., Sánchez-Navalón, B. y Garrote-Rojas, D. (2016). Educación inclusiva en contextos inéditos: la implementación de la Pedagogía Hospitalaria. Revista Latinoamericana de Ciencias Sociales, Niñez y Juventud, 14(2), 1507-1522. https://doi.org/10.11600/1692715x.14242240815

Pellicano, L., Bölte, S. y Stahmer, A. (2018). The current illusion of educational inclusion. In Autism (Vol. 22, Issue 4, pp. 386-387). SAGE Publications Ltd. https://doi.org/10.1177/1362361318766166

Pereiro González, M. del C. y Penas Busto, Y. (2017). Pensamiento computacional: creación y desarrollo de aprendizajes y conocimientos reales desde una perspectiva inclusiva y de compensación de las desigualdades. Revista de Estudios e Investigación En Psicología y Educación, Extr.(13), 347-351. https://doi.org/10.17979/reipe.2017.0.13.3228

Peterson-Ahmad, M. B., Stepp, J. B. y Somerville, K. (2018). Teaching Pre-Service Teachers How to Utilize Web 2.0 Platforms to Support the Educational Needs of Students with Disabilities in General Education Classrooms. Education Sciences, 8(2), 1-9. https://doi.org/10.3390/educsci8020080

Polaino Lorente, A. y Lizasoain, O. (1992). La pedagogía hospitalaria en Europa: la historia recietne de un movimiento pedagógico innovador. Psicothema, 4(1), 49-67.

Roberts-Yates, C. y Silvera-Tawil, D. (2019). Better Education Opportunities for Students with Autism and Intellectual Disabilities through Digital Technology. International Journal of Special Education, 34(1), 197-210.

Saladino, M., Marin Suelves, D. y San Martín Alonso, Á. (2020). Percepción docente del aprendizaje mediado tecnológicamente en aulas italianas. Revista Interuniversitaria de Formación Del Profesorado. Continuación de La Antigua Revista de Escuelas Normales, 34(3). https://doi.org/10.47553/rifop.v34i3.80593

Salgado Bocanegra, C. (2020). Miradas de la pedagogía hospitalaria: reflexiones encarnadas de un docente hospitalario. Infancias Imágenes, 19(1), 100-107. https://doi.org/10.14483/16579089.14684 
Sani-Bozkurt, S., Vuran, S. y Akbulut, Y. (2017). Design and Use of Interactive Social Stories for Children with Autism Spectrum Disorder (ASD). Contemporary Educational Technology, 8(1), $1-25$.

Serrano Sánchez, J. L. y Castañeda Quintero, L. J. (2016). Proyecto EDUMOBSPITALARIOS: desarrollo profesional docente e innovación con m-learning en aulas hospitalarias. EDUTEC. Revista Electrónica de Tecnología Educativa, 55, 0-3.

Solovieva, O. V., Palieva, N. A., Borozinets, N. M., Kozlovskaya, G. Y. y Prilepko, J. V. (2020). Development of Digital Intelligence Among Participants of Inclusive Educational Process. $\begin{array}{llll}\text { Propositos } y & \text { Representaciones, } & \text { 8(SPE(2)), }\end{array}$ https://doi.org/10.20511/pyr2020.v8nSPE2.675

Tumino, M. C. y Bournissen, J. M. (2017). Una mirada a la integración de los valores desde el rol de las TIC. Ciencia, Docencia y Tecnología, 28(54), 188-215.

UNESCO. (2015). Replantar la educación: ¿Hacia un bien común mundial? Ediciones UNESCO.

UNESCO. (2019). Compromiso de Cali sobre Equidad e Inclusión en la Educación.

Urrútia, G. y Bonfill, X. (2010). PRISMA declaration: A proposal to improve the publication of systematic reviews and meta-analyses. Medicina Clinica, 135(11), 507-511. https://doi.org/10.1016/j.medcli.2010.01.015

Van Dijk, J. (2008). Digital divide in Europe. In A. Chadwick \& P. Howard (Eds.), The handbook of Internet Policies. Routledge.

Vaquero Tió, E., Marco, I., Paola, M. y Ángels, B. M. (2016). Una revisión de la literatura sobre el uso de las TIC en el ámbito de la intervención sociofamiliar. In R. Roig-Vila (Ed.), Tecnología, innovación e investigación en los procesos de enseñanza-aprendizaje (pp. 1919-1928). Octaedro.

Vico Linde, P. (2019). Percepción docente conforme al uso de los recursos digitales en la pizarra digital interactiva con alumnos de Necesidades Educativas Especiales. EDUTEC. Revista Electrónica de Tecnología Educativa, Septiembre(69), 121-138. https://doi.org/10.21556/edutec.2019.69.1295

Violant Holz, V., Molina Garuz, M. C. y Pastor Vicente, C. (2011). Pedagogía Hospitalaria. Bases para una atención integral. Laertes $\mathrm{S}$.

\section{Para citar este artículo:}

García-Parra, M., y Pérez Sepulcre, M. (2021). Vínculos entre Ética, Tecnología Educativa y Pedagogía Hospitalaria: una revisión sistematizada de la literatura. Edutec. Revista Electrónica De Tecnología Educativa, (77), 17-34. https://doi.org/10.21556/edutec.2021.77.2121 